\title{
Changes of Hypocretin (Orexin) System in Schizophrenia: From Plasma to Brain
}

\author{
Jing Lu ${ }^{1,2}$, Man-Li Huang ${ }^{1,2}$, Jin-Hui Li ${ }^{3}$, Kang-Yu Jin' ${ }^{1}$, Hai-Mei Li', Ting-Ting Mou ${ }^{1,2}$, Rolf Fronczek ${ }^{4, \oplus, ~ J i n-F e n g ~ D u a n ~}{ }^{1,2}$, \\ Wei-Juan Xu1 ${ }^{1,2}$, Dick Swaab ${ }^{5,6}$, and Ai-Min Bao ${ }^{1,2,6}$ \\ ${ }^{1}$ Department of Psychiatry, the First Affiliated Hospital, Zhejiang University School of Medicine, Hangzhou, China; ${ }^{2}$ The Key \\ Laboratory of Mental Disorder Management in Zhejiang Province, Hangzhou, China; ${ }^{3}$ Department of Traditional Chinese Medicine \\ \& Rehabilitation, the Second Affiliated Hospital of Zhejiang University School of Medicine, Hangzhou, China; ${ }^{4}$ Department of \\ Neurology, Leiden University Medical Center, Leiden, Netherlands; ${ }^{5}$ Netherlands Institute for Neuroscience, an Institute of the \\ Royal Netherlands Academy of Arts and Sciences, Amsterdam, The Netherlands; ${ }^{6}$ NHC and CAMS Key Laboratory of Medical \\ Neurobiology, School of Brain Science and Brain Medicine, Zhejiang University School of Medicine, Hangzhou, China \\ *To whom correspondence should be addressed; Department of Psychiatry, the First Affiliated Hospital, Zhejiang University School of \\ Medicine, Hangzhou, China; tel: +86 571 88208789, fax: +86 571 88208094, e-mail: baoaimin@zju.edu.cn
}

Hypocretin (also called orexin) regulates various functions, such as sleep-wake rhythms, attention, cognition, and energy balance, which show significant changes in schizophrenia (SCZ). We aimed to identify alterations in the hypocretin system in SCZ patients. We measured plasma hypocretin-1 levels in SCZ patients and healthy controls and found significantly decreased plasma hypocretin-1 levels in SCZ patients, which was mainly due to a significant decrease in female SCZ patients compared with female controls. In addition, we measured postmortem hypothalamic hypocretin-1-immunoreactivity (ir), ventricular cerebrospinal fluid (CSF) hypocretin-1 levels, and hypocretin receptor (Hcrt-R) mRNA expression in the superior frontal gyrus (SFG) in SCZ patients and controls We observed a significant decrease in the amount of hypothalamic hypocretin-1 ir in SCZ patients, which was due to decreased amounts in female but not male patients. Moreover, Hcrt-R2 mRNA in the SFG was decreased in female SCZ patients compared with female controls, while male $\mathrm{SCZ}$ patients showed a trend of increased Hcrt-R1 mRNA and Hcrt-R2 mRNA expression compared with male controls. We conclude that central hypocretin neurotransmission is decreased in SCZ patients, especially female patients, and this is reflected in the plasma.

Key words: schizophrenia/hypocretin/orexin, hypocretin receptor/cerebrospinal fluid/hypothalamus/superior frontal gyrus/plasma

\section{Introduction}

Schizophrenia (SCZ) is a severe mental disorder with a heterogeneous combination of symptoms. These symptoms can be divided into "positive," "negative," and "cognitive" categories. Negative symptoms include anhedonia, hypobulia, social withdrawal, and lack of motivation, while positive symptoms include hallucinations and delusions. ${ }^{1}$ The pathogenesis of SCZ is proposed to be a combination of genetic and environmental factors that result in disordered neurocircuits mediated by neurotransmitters and neuromodulators.

Among these key pathogenic molecules, hypocretin (orexin) caught our attention. Hypocretinergic neurons are exclusively located in the hypothalamus, ${ }^{2}$ producing 2 types of hypocretin: hypocretin-1, which is the major type in the brain, and hypocretin-2. These neurons send inputs to many brain areas, including the hypothalamus, prefrontal cortex (PFC), hippocampus, dorsal raphe nucleus, and locus coeruleus, ${ }^{3}$ influencing functions via 2 types of receptors, hypocretin receptor-1 and -2 (Hcrt-R1 and Hcrt-R2). Hcrt-R1 and Hcrt-R2 are both G proteincoupled receptors that are widely expressed throughout the central nervous system. Although the majority of human Hcrt-R variants are only weakly associated with diseases including SCZ, other disorders in hypocretine mechanisms such as disorders in the expression of hypocretin and its receptors, or functional interactions and/ or heterodimerization between wild-type and variant Hcrt-Rs and opioid or cannabinoid receptors may still be of potential pharmacogenetic significance. ${ }^{4}$ Hypocretin regulates physiological functions, such as the sleep-wake cycle, attention, cognition, and energy balance, all of which are severely altered in SCZ. ${ }^{5}$ And narcoleptic patients, who are characterized by a loss of hypocretin, ${ }^{6}$ are sometimes misdiagnosed with SCZ due to their hypnagogic hallucinations. ${ }^{7}$

Although several clinical studies have reported changes in plasma hypocretin-1 levels in SCZ, their findings appear 
equivocal. Some studies showed that elevated plasma hypocretin-1 levels in SCZ patients were related to negative and disorganized symptoms, $, 8,8$ or obesity, ${ }^{10}$ whereas a meta-analysis showed no difference in plasma hypocretin-1 levels between SCZ and controls. ${ }^{11}$ In addition, a disordered plasma hypocretin-1 circadian rhythm of was found in SCZ patients, with a peak phase delay of 4 hours. ${ }^{8}$ It should be noted, however, that the patients studied in these experiments were all undergoing medical treatment, and antipsychotic treatment has been found to reduce plasma hypocretin-1 levels. ${ }^{12,13}$ It is, therefore, important to determine plasma hypocretin levels in drug-naive patients.

To date, the possibility of a disordered hypocretin system in the brain in SCZ has not been studied; thus, questions remain regarding whether and how the observed changes in plasma hypocretin-1 may reflect hypocretin system changes in the brain of SCZ patients. In addition, the hypocretin system was found to play an important, partly sex-dependent, regulatory role in social (fear) behavior ${ }^{14}$ and regulates in a sexually dimorphic way cognitive flexibility. ${ }^{15}$ In addition, the SCZ disease process itself shows sex differences, manifested as an earlier age of onset for the first episode in males, which is $18-25$ years, and approximately 3 years ahead of females. ${ }^{16}$ Moreover, sex differences in gene expression and clinical manifestations were also found in SCZ. ${ }^{16,17}$ Therefore, we paid special attention to the potential sex differences in the hypocretin system changes in this disease. ${ }^{18}$

Based upon the evidence mentioned above, in the present study, we first measured the changes in the plasma hypocretin-1 levels of clinical SCZ patients who were in their first episode and medicine-naive. Second, from postmortem brain material, we determined the amount of hypocretin-1 immunoreactivity (ir) at the hypothalamic production site, and the hypocretin-1 levels in cerebrospinal fluid (CSF). In addition, we determine the mRNA expression of Hcrt-R1 and Hcrt-R2 in the superior frontal gyrus (SFG), a brain area that shows significant structural and cognitive changes in SCZ. ${ }^{19}$ This brain area was selected, for instance, because decreased cortical thickness, oligodendrocytes and white matter of SFG were observed in SCZ patients in relation to decreased cognitive control and role functioning, ${ }^{20,21}$ while a higher regional homogeneity in the left medial SFG was found in fMRI in relation to delusional symptoms of SCZ.22

\section{Methods}

\section{Clinical Study}

SCZ Patients and Healthy Controls. In total, 61 firstepisode, medicine-naive SCZ patients (27 men, aged $24.2 \pm 10.1$ years (mean $\pm \mathrm{SD}$ ); and 34 women, aged $28.2 \pm 11.3$ years) were recruited from the Department of Psychiatry, the First Affiliated Hospital of Zhejiang University School of Medicine. Informed consent was obtained from each patient or healthy control upon participation. The criteria for recruitment of SCZ patients were as follows: (1) aged 18-65 years, (2) right-handed, (3) Han Chinese ethnicity, (4) diagnosed with SCZ according to the Diagnostic and Statistical Manual of Mental Disorders IV (DSM-IV), (5) having attended at least 9 years of formal education. The 82 healthy control subjects ( 38 men, aged $27.6 \pm 9.3$ years and 44 women, aged $27.1 \pm 9.6$ years) underwent their yearly physical examination in the same hospital and voluntarily participated in this study during the same period. The exclusion criteria for the participants were as follows: (1) abnormal body mass index (BMI; BMI < 18.5 or BMI > 24), (2) history of neurological or psychiatric disorders (except SCZ) and MRI (Magnetic Resonance Imaging) evidence of structural brain abnormalities, (3) taking medication or substance abuse. There were no significant differences in the age, sex ratio, or education years between SCZ and control subjects $(P \geq .37)$. The study was carried out in accordance with the latest version of the Declaration of Helsinki. All participants signed informed consent forms and the study was approved by the Medical Ethics Committee of the First Affiliated Hospital of Zhejiang University School of Medicine.

Blood Sample Collection and Plasma Hypocretin-1 Level Measurement. Venous blood samples were collected between 06:00 a.m. and 09:00 a.m., following a fasting period of 8-12 hours and assayed for hypocretin-1 levels (supplementary material).

\section{Postmortem Human Brain Material Study}

In total, 56 human postmortem samples were studied, including 10 hypothalami, 7 CSF samples, and 11 SFG specimens of SCZ patients, and the same number of respectively well-matched controls. All postmortem human brain materials were obtained from the Netherlands Brain Bank (NBB). Informed consent for brain autopsy, and for the use of the brain material and medical records for research purposes, was given by the donor or their next of kin. SCZ patients and controls were matched for possible confounding factors including age, sex, postmortem interval (PMI), fixation time, clock time of death, month of death, CSF-pH (a measure of agonal state), brain weight, and Braak stage of Alzheimer's pathology. ${ }^{23}$ Clinicopathological details and $P$-values for matching are shown in table 1 and supplementary tables 1-3. Diagnosis of SCZ was made during life and confirmed according to DSM-IV by qualified psychiatrists using the extensive medical records of the NBB, which also contained welldocumented diagnoses and the year of onset of SCZ from psychiatric clinics. Exclusion criteria were the presence of any other neurological or psychiatric diseases or major neuropathological abnormalities.

Immunocytochemistry of Hypothalamic Hypocretin-1 ir. Hypothalami were fixed in $10 \% \mathrm{PBS}(\mathrm{pH} 7.4)$ formalin 


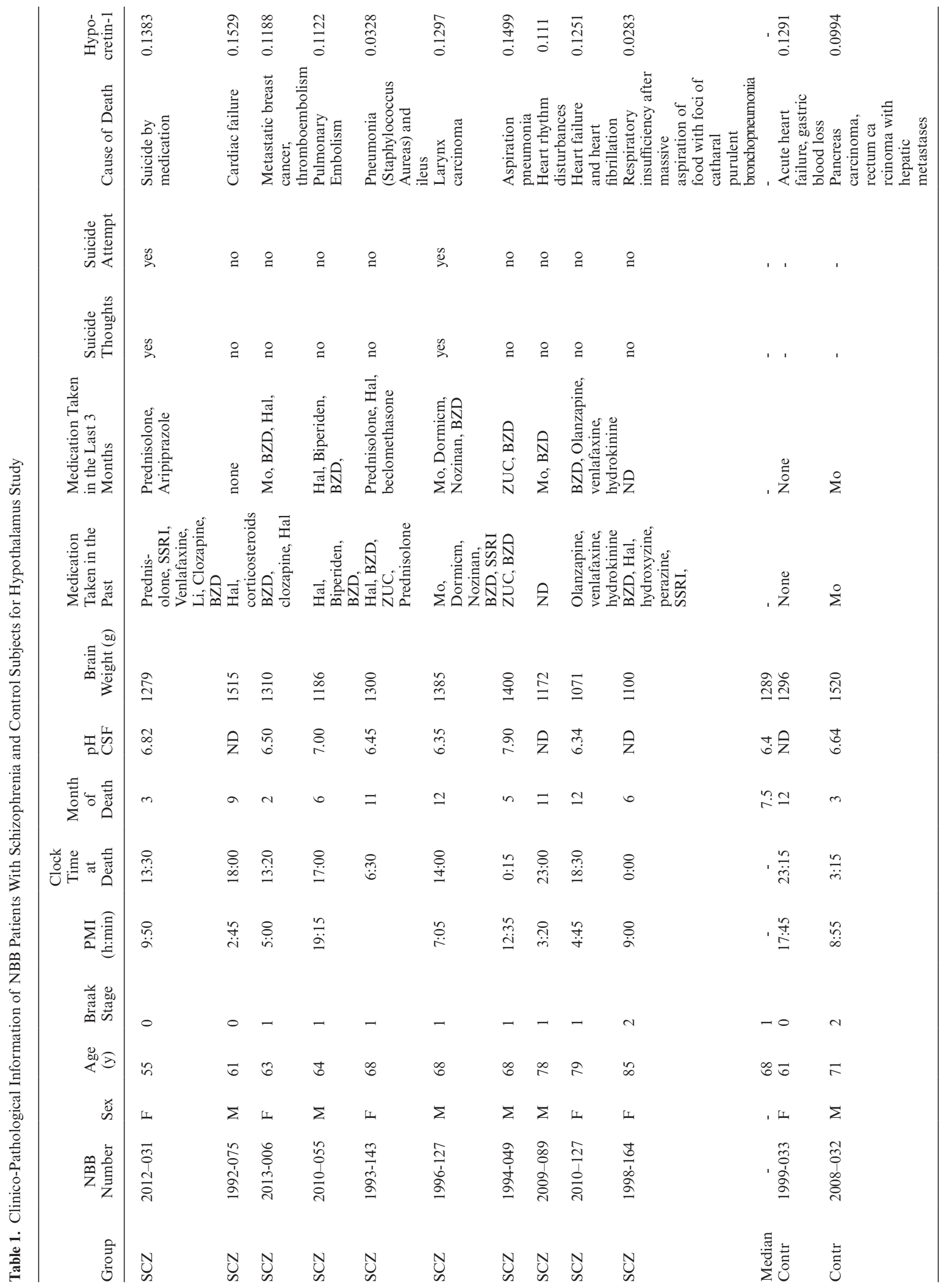




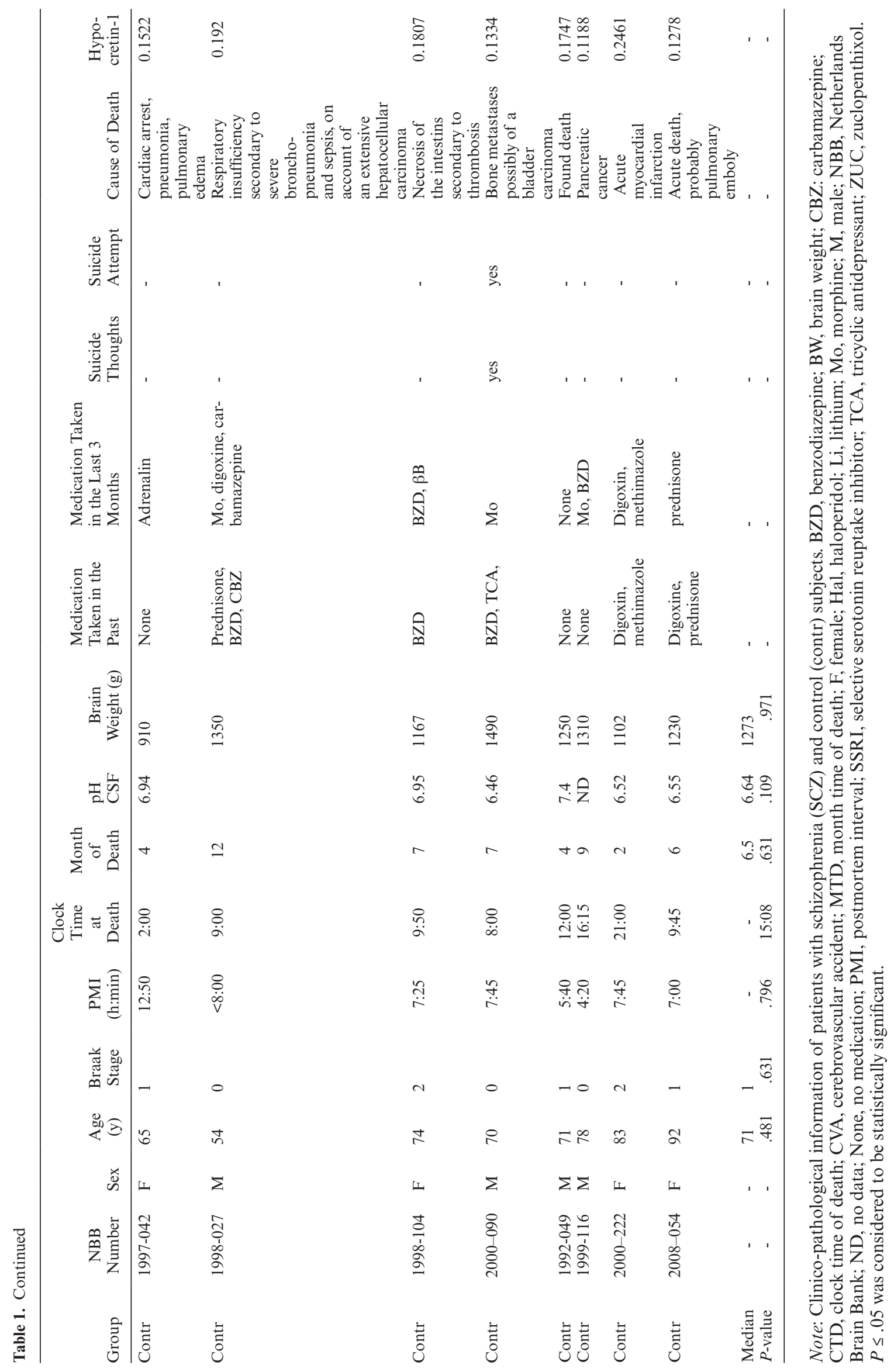


at room temperature, and were paraffin-embedded and serially-sectioned at $6 \mu \mathrm{m}$ in the rostro-caudal direction. Every 100th section in the expected hypocretin cell area was stained using a hypocretin-1 antibody (catalog no. H-003-30; Phoenix Pharmaceuticals, Inc.) at a 1:20,000 dilution. The specificity of the antibody has been confirmed in our previous studies. ${ }^{24,25}$ Hypocretin-1 ir was quantified by an image analysis procedure described extensively in our previous studies ${ }^{25}$ (supplementary material).

MeasurementofVentricularCSFHypocretin-1Levels. Ventricular CSF was collected during autopsy, centrifuged at $2500 \mathrm{rpm}$ for 10 minutes and the supernatant was immediately stored at $-80^{\circ} \mathrm{C}$ until further use. CSF hypocretin-1 levels were measured using a commercially available radioimmunoassay (RIA) kit (Phoenix Pharmaceuticals) (supplementary material).

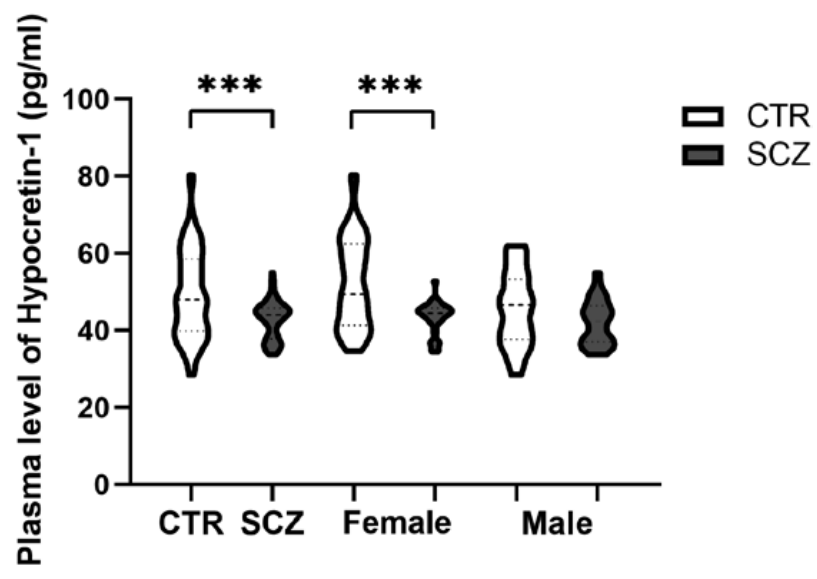

Fig. 1. Plasma level of Hypocretin-1 between control (CON, $n=82$ ) and schizophrenia (SCZ, $n=61$ ) group on the left side, and for females group comparison (ie, female CON: $n=44$; female SCZ: $n=34$ ) in the middle, or males group comparison (ie, male CON: $n=38$; male SCZ: $n=27$ ) in the right.
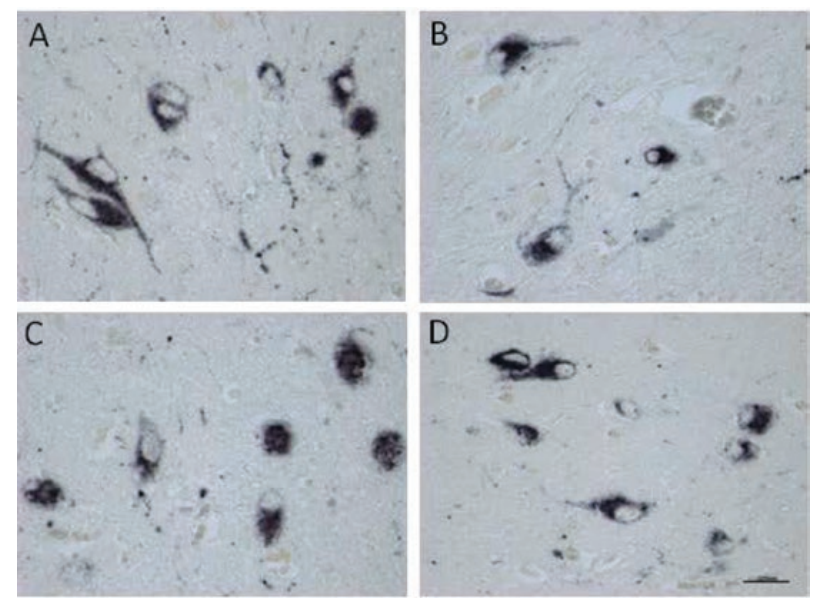

Fig. 2. Examples of staining of hypocretin-1 cells bodies in the lateral hypothalamus of (A) a female control (NBB\#2000-040). (B) a female schizophrenia patient (NBB\#2010-127). (C) a male control (NBB\#2010-055). (D) a male schizophrenia patient (NBB\#s922-261). Bar $=0.025 \mathrm{~mm}$. There was no significant difference in the intensity of staining and the distribution pattern.
Quantitative PCR (qPCR) of Hypocretin Receptor mRNA in the $S F G$. SFG cryostat sections of $50-\mu \mathrm{m}$ thickness were cut from the left side of the cortex and the gray matter containing all 6 layers was isolated as described previously. ${ }^{26}$ Information regarding the selection of Hcrt-R1 and Hcrt-R2, and the reference genes together with the primer sequences, are shown in supplementary table 3 . RNA isolation, cDNA synthesis,

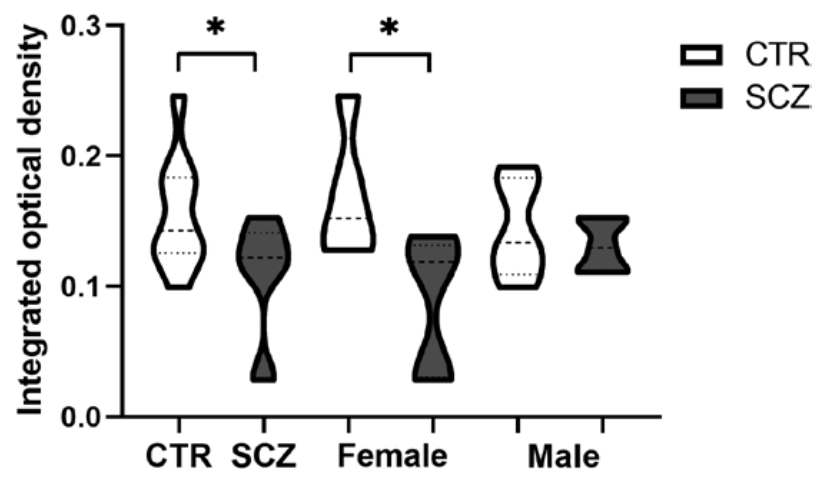

Fig. 3. Comparison of the integrated optical density (IOD) of hypocretin-1 between the entire control $(\mathrm{CON}, n=10)$ and the entire schizophrenia group (SCZ, $n=10)$, the female CON $(n=5)$ and female SCZ group $(n=5)$, and the male $\mathrm{CON}(n=5)$ and male SCZ group $(n=5)$.
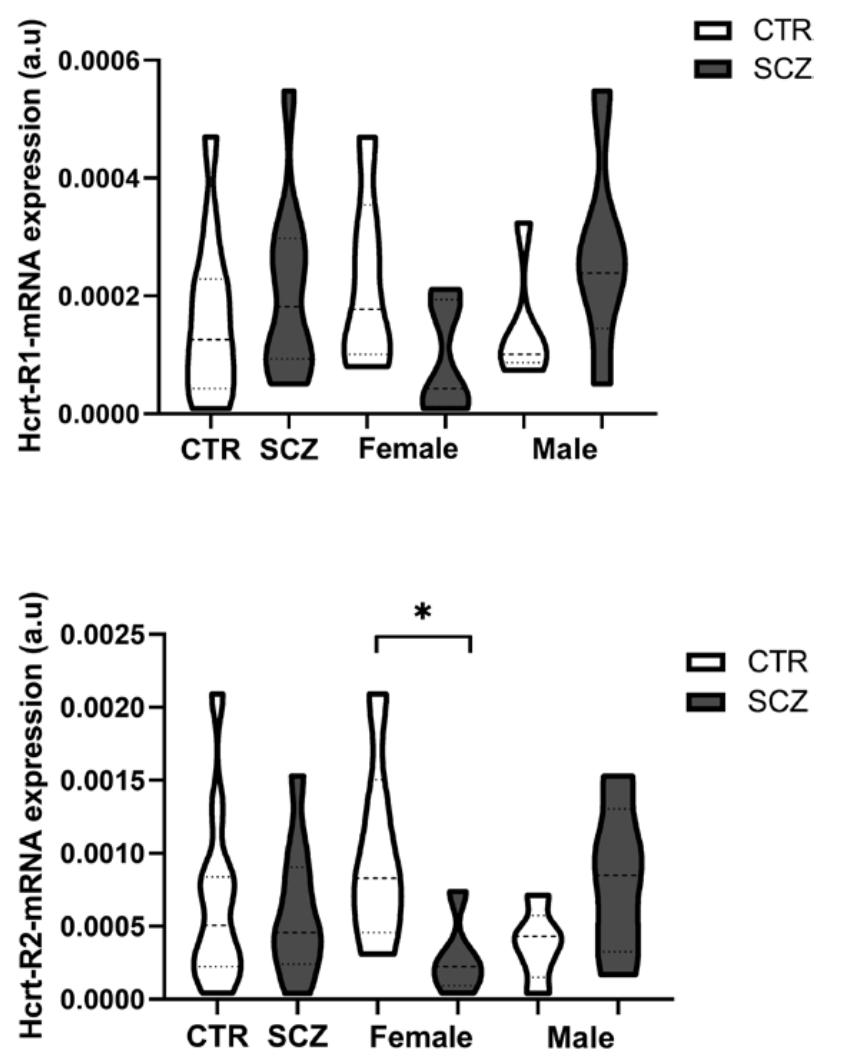

Fig. 4. Column showing the Hcrt-receptors-mRNA expression in the superior frontal gyrus in the entire schizophrenia $(n=11)$ and control group $(n=11)$ and in the female CTR $(n=6)$ and female SCZ group $(n=6)$, and the male CTR $(n=5)$ and male SCZ group $(n=5)$. 
and $\mathrm{qPCR}$ were performed as described previously. ${ }^{25,27}$ In addition, a normalization strategy was used to remove sampling-related differences in RNA quantity. ${ }^{25}$

Relationship Between Hypocretin System Changes and SCZ Symptoms. The levels of plasma hyppocretin-1 levels, hypothalamic hypocretin-1 ir, CSF hypocretin, and SFG Hcrt-R-mRNA were related to the presence of hallucination, suicide ideations, inactivity, disturbed attention, paranoid psychosis, mental retardation, anxiety, depressive symptoms, and mood swings. Only the significant findings are reported in the Results section.

Statistics. Since all the experimental data were normally distributed, as confirmed by Kolmogorov-Smirnov tests of normality, the Student's t-test was used to analyze the differences between 2 groups, and 2-way ANOVA was applied using SCZ and sex as the main factors. Correlations were examined using the Pearson's test. Differences in clock time and month of death were analyzed using the Mardia-Watson-Wheeler test. All tests were 2-tailed. $P \leq$ .05 was considered significant and $.05<P \leq .1$ was considered to indicate a trend. Statistical analyses were carried out using SPSS Statistics ver. 20 (SPSS, Inc.).

\section{Results}

\section{Plasma Hypocretin-1 Levels in SCZ Patients}

The SCZ group $(n=61)$ showed significantly lower plasma hypocretin-1 levels than the healthy control group ( $n=82, P<.0001$, figure 1$)$. In addition, female SCZ patients $(n=34)$ had significantly lower plasma hypocretin-1 levels than female controls $(n=44, P=.001$, figure 1$)$, while only a trend was apparent for lower plasma hypocretin-1 levels in the male SCZ patients $(n=27)$ compared with male controls $(n=38, P=.094)$. Two-way ANOVA revealed that the effect of $\mathrm{SCZ}$ on plasma hypocretin-1 and the effect of sex on plasma hypocretin-1 remained significant (supplementary table 4).

Within the control group, females $(n=44,52.42 \pm$ $11.94 \mathrm{pg} / \mathrm{ml}$ ) showed significantly higher plasma hypocretin-1 levels than males $(n=38,45.58 \pm 9.63, P=.007$, figure 1); while within the SCZ group, there was no significant difference between the male $(n=27,42.12 \pm$ 5.82) and female patients $(n=34,43.3 \pm 4.09, P=.342$; figure 1). There was a significant negative correlation between age and plasma hypocretin-1 levels in the control group $(r=-.251, P=.023)$, and a significant correlation was found in female controls between age and hypocretin-1 level ( $r=-.390, P=.009)$, although not in male controls $(r=-.059, P=.023)$. This correlation was also absent in the SCZ group $(r=.096, P=.462)$.

\section{Hypocretin-1 ir in the Hypothalamus}

The location and staining intensity of the hypocretin-1stained neurons were in agreement with the results that we have described previously, ${ }^{25}$ and similar in controls and SCZ patients (figures $2 \mathrm{~A}-\mathrm{D}$ ). The integrated optical density (IOD) of hypocretin-1 ir was significantly lower in the SCZ group compared with the control group $(P=.032, n=10$, figure 3$)$. This difference appeared to be due to the female $\mathrm{SCZ}$ patients, who showed significantly lower levels than the female controls $(P=.042, n=5)$. Two-way ANOVA revealed that the effect of SCZ on hypothalamic hypocretin-1 ir remained significant (supplementary table 4).

There was no significant correlation between the putative confounding factors age, PMI, brain weight, fixation time, or CSF-pH, on hypocretin-1 ir either in controls ( $P$ $\geq .116)$ or in the SCZ group $(P \geq .712)$. Moreover, there was no significant correlation between the duration of SCZ and hypocretin-1 ir $(P=.668, r=.167)$.

\section{Hypocretin-1 Levels in Ventricular CSF}

CSF hypocretin-1 levels in SCZ patients were not different from those in controls $(P=.145, n=7)$. However, there was a trend $(P=.079)$ toward a reduction in CSF hypocretin-1 levels in female SCZ patients $(n=5)$ compared with female controls. No comparison of CSF hypocretin-1 levels was made between the male SCZ patients $(n=2)$ and male controls $(n=2)$ because of limited samples.

There was a significant negative correlation between age and CSF hypocretin-1 levels in the control group $(P=.041, r=-.775, n=7)$, but not in SCZ patients $(P=.496, r=-.312, n=7)$. In addition, there was no significant correlation between the duration of SCZ and CSF hypocretin-1 levels $(P=.173, r=-.579)$.

\section{Hcrt-R $m$ RNA Expression in the SFG}

There was no significant difference in Hcrt-R1 $(P=.450)$ and Hcrt-R2 $(P=.693)$ mRNA expression in the SFG between the SCZ and control groups. However, Hcrt-R2 mRNA levels showed a trend $(P=.050$, figure 4$)$ toward a decrease in female $\mathrm{SCZ}$ patients compared with female controls, whereas both Hcrtr-R1 and -2 mRNA levels showed trends (both $P=.079$ ) toward an increase in male SCZ patients compared with male controls. Two-way ANOVA revealed that there was a significant interaction between sex and SCZ in Hcrt-R1 and Hcrt-R2 mRNA expression (figure 4, supplementary table 4).

\section{Discussion}

The present study shows, for the first time, decreased activity of the hypocretin system in SCZ patients, manifesting both as decreased plasma hypocretin-1 levels in medicine-naive clinical SCZ patients and in hypocretin-1 ir in postmortem hypothalamus. In addition, a difference was found in the diminished activity of hypocretin system in SCZ between the sexes. These 
differences were more obvious in female patients than in males, again manifesting both in the plasma hypocretin-1 levels of clinical SCZ patients and in postmortem hypothalamus and CSF samples. On the contrary, the trends toward increased Hcrt-R1 and Hcrt-R2 mRNA expression in male SCZ SFG imply the possibility of slightly increased sensitivity to hypocretin in male SCZ patients. Our data thus support a difference between the sexes in terms of alterations in the hypocretin system in SCZ.

To our knowledge, decreased plasma hypocretin-1 levels in medicine-naive SCZ patients during their first episode are a novel finding. Liu group found higher plasma hypocretin levels in obese SCZ patients and proposed that hypocretin might be a factor affecting the BMI of SCZ patients. ${ }^{10}$ In addition, another study showed SCZ patients had significantly higher plasma hypocretin-1 levels than healthy controls. However, the finding that SCZ patients with high $(>80 \mathrm{pg} / \mathrm{ml})$ hypocretin levels had significantly fewer negative and disorganized symptoms than the patients with low $(<80 \mathrm{pg} / \mathrm{mL})$ hypocretin levels was proposed to be related to the fact that all the SCZ patients in their study were taking antipsychotic treatments. ${ }^{5}$ Indeed, a previous study showed that $\mathrm{SCZ}$ patients who were using Clozapine had higher plasma hypocretin-1 than controls, ${ }^{9}$ and other studies have shown that antipsychotic treatment may reduce plasma hypocretin-1 levels. ${ }^{12,13}$ These data show the importance of studying the hypocretin changes in medicine-naive SCZ patients with normal BMI. Our findings of decreased plasma hypocretin raises the questions what the function may be of circulating hypocretin and whether diminished circulating hypocretin may have functional implications. In the first place, it should also be noted that hypocretin can quickly pass the blood-brain barrier by simple diffusion, ${ }^{28}$ so that circulating hypocretin levels may just reflect central hypocretin level changes. ${ }^{29}$ This gives the hope to further study the possibility of using blood hypocretin levels as a biological marker for changes in central hypocretin levels for instance in SCZ. Moreover, recent studies have demonstrated possible functions of peripheral hypocretin. For instance, hypocretin in the enteric system may protect against systemic inflammation and in the brain against central inflammation. Indeed, intranasal administration of exogenous hypocretin-1 showed neuroprotective effects and inhibited central inflammation in animals. ${ }^{30}$ Furthermore, peripheral hypocretin in the intestinal mucosa modulates brain functions, indicating the presence of a possible hypocretinergic gut-brain network..$^{31,32}$ The role of the lower circulating hypocretin levels we observed deserves thus further study in SCZ, eg, in relation to inflammatory processes. The distribution of hypocretin-1 ir in the cytoplasm and fibers of hypothalamic neurons of both the SCZ patients and controls was the same as that found in our previous study on depression. ${ }^{25}$ In addition, the diminished activity of the hypocretin system in SCZ brain, especially female brains, is supported by the plasma study. Postmortem ventricular CSF hypocretin-1 levels showed no significant differences between male SCZ and male controls, which in agreement with a previous study that showed that hypocretin-1 levels in clinical lumbar puncture CSF were not different between male SCZ patients and male controls. ${ }^{33}$ Moreover, Gerashchenko et al. found that lesioning $70 \%$ of the hypocretin neurons in rats resulted in an approximately $50 \%$ decline in $\mathrm{CSF}$ hypocretin levels. ${ }^{34}$ This finding agrees well with our observation that an approximately $47 \%$ decrease in hypothalamic hypocretin-1 ir resulted only in a downward trend in ventricular CSF hypocretin-1 levels, although the small number of CSF samples, especially of the male SCZ group was a limitation of present study. Plasma hypocretin-1 levels might reflect CSF levels to some extent. ${ }^{29}$ In control subjects, we indeed found a negative correlation with age for both in vivo-obtained plasma and postmortem CSF hypocretin-1, which is consistent with a study showing an age-related decline in the number of hypothalamic hypocretin neurons in the range from 0 to 60 years in control subjects. ${ }^{2}$ It should also be noted that the mRNA samples of SFG used in our study did not allow an analysis of cellular content as performed by Karpiński et al. (2020), ${ }^{35}$ who found the analysis of gene expression in SCZ patients might be confounded by this parameter. Future studies should include this parameter. Our results convincingly show that the activity of the hypocretin system is diminished in female SCZ patients, both through our clinical and postmortem studies. It was, for instance, recently found hypocretin deficiency selectively impaired female rats' performance on attentional set shifting task, ${ }^{15}$ which fits with the sex difference we found in hypocretin changes in SCZ. Some sex differences in signs and symptoms in SCZ patients may, at least partly, be related to the sex-dependent hypocretin changes we observed. Hypocretin-1 has been proposed to have a protective effect against the development of metabolic syndrome in SCZ patients receiving antipsychotic treatment, ${ }^{9}$ and the worse lipid metabolic dysfunction found in female patients ${ }^{36}$ is in agreement with our findings indicating an altered hypocretin system. In addition, it has been reported that male patients have an earlier disease onset, ${ }^{37}$ more negative symptoms, and poorer functioning, ${ }^{38}$ while female patients show a higher prevalence of the paranoid subtype, more severe positive symptoms, more depressive symptoms, and fewer negative symptoms. ${ }^{18}$ It should be noted that some researchers have proposed that, overall, women are more likely to express affective symptoms than men, and the sex differences in symptom expression in SCZ may thus be related to sex (with respect to illness expression) rather than to $\mathrm{SCZ}$ proper. ${ }^{39} \mathrm{An}$ 
analysis of psychotropic drug prescriptions in Asia also showed that SCZ women have more delusions and hallucinations than men. ${ }^{40}$ Hypocretin was found to excite thalamic neurons and their projections to the PFC, which regulate attention and awareness, ${ }^{41}$ while reduced connectivity between the midline-intralaminar thalamic nuclei and the PFC has been proposed to contribute to cognitive deficits in SCZ. ${ }^{42}$ In addition, although Misiak (2019) in a meta-analysis found no significant changes in hypocretin plasma levels in first-episode psychosis patients, ${ }^{11}$ Lis et al's review indicated that altered hypocretin regulation of appetite was observed in early psychosis, and impairments of appetite-regulating hormones may attributable to intrinsic disease mechanisms and be affected by atypical antipsychotics. ${ }^{43}$ Hypocretin is a neuropeptide crucially involved in energy balance ${ }^{44}$ while impaired appetite regulation was observed in early SCZ and before medical treatment, and, moreover, many $\mathrm{SCZ}$ patients have energy metabolism disturbance. ${ }^{11}$ It deserves, therefore, further future study on the involvement of hypocretin in the impaired appetite regulation in SCZ, especially its sex differences. Finally, it is remarkable that the alterations in the hypocretin system that we previously found in mood disorders are exactly the opposite of those we observed in the present study's SCZ patients, ${ }^{25}$ while neither of them finds significant sex differences in the hypocretin systems in control subjects. A recent study reported that hypocretin might be important in the etiology of stress-related psychiatric disorders that present differently in men and women. Thus, targeting hypocretin could potentially ameliorate various phenotypes of stress-related illness in a sex-specific way. ${ }^{45}$

One of the inherent potential confounding factors in a postmortem study is medication use. Indeed, animal studies have shown that the medication used by the SCZ patients in the present study, such as benzodiazepines, ${ }^{46}$ haloperidol, ${ }^{47}$ and fluoxetine ${ }^{48}$ may inhibit hypocretin neurons and/or decrease hypocretin levels. In addition, a study reported that CSF hypocretin-1 levels were significantly lower in patients treated with haloperidol than in untreated patients. ${ }^{47}$ Concerning the postmortem observation, we do not believe that our main conclusions are confounded by antipsychotic medicines since decreased hypothalamic hypocretin- 1 ir was only observed in female SCZ patients, while all SCZ patients in the hypothalamus study had used antipsychotic medication. Furthermore, in our hypothalamus study, there was no significant difference in hypocretin-1 ir between patients who took either one of these 3 medications compared with patients that did not use them $(P=.667)$. Moreover, in the SFG study, neither Hcrt-R1 mRNA $(P=.766)$ nor Hcrt-R2 mRNA $(P=.255)$ showed a difference between SCZ with or without these 3 medicines. Finally, our findings of decreased plasma hypocretin levels in medicine-naive SCZ patients, especially female patients, supported our postmortem conclusion.

\section{Conclusion}

The activity of the hypocretin system was decreased in SCZ patients, especially female patients. The relationship between this change and particular symptoms of SCZ requires further future elucidation. In addition, our findings indicate that if, in the future, hypocretin agonists or antagonists ${ }^{49}$ were to have a place in SCZ treatment, the sex of patients should be taken into consideration.

\section{Supplementary Material}

Supplementary material is available at Schizophrenia Bulletin online.

\section{Funding}

This study was supported by the National Key R\&D Program of China (2016YFC1306700), the National Natural Science Foundation of China (91332102, 81801340, and 31271130).

\section{Acknowledgments}

The authors are grateful to the Netherlands Brain Bank (Director Dr. Inge Huitinga) for providing human brain material and clinical details, and to Stichting Vrienden from the Dutch Brain Institute for their support. The authors have declared that there are no conflicts of interest in relation to the subject of this study.

\section{References}

1. Kahn RS, Sommer IE, Murray RM, et al. Schizophrenia. Nat Rev Dis Primers. 2015;1:15067.

2. Hunt NJ, Rodriguez ML, Waters KA, Machaalani R. Changes in orexin (hypocretin) neuronal expression with normal aging in the human hypothalamus. Neurobiol Aging. 2015;36(1):292-300.

3. Peyron C, Tighe DK, van den Pol AN, et al. Neurons containing hypocretin (orexin) project to multiple neuronal systems. J Neurosci. 1998;18(23):9996-10015.

4. Thompson MD, Sakurai T, Rainero I, Maj MC, Kukkonen JP. Orexin receptor multimerization versus functional interactions: neuropharmacological implications for opioid and cannabinoid signalling and pharmacogenetics. Pharmaceuticals (Basel) 2017;10(4):1-20.

5. Chien YL, Liu CM, Shan JC, et al. Elevated plasma orexin A levels in a subgroup of patients with schizophrenia associated with fewer negative and disorganized symptoms. Psychoneuroendocrinology. 2015;53:1-9. 
6. Peyron C, Faraco J, Rogers W, et al. A mutation in a case of early onset narcolepsy and a generalized absence of hypocretin peptides in human narcoleptic brains. Nat Med. 2000;6(9):991-997.

7. Douglass AB. Narcolepsy: differential diagnosis or etiology in some cases of bipolar disorder and schizophrenia? CNS Spectr. 2003;8(2):120-126.

8. Sun HQ, Li SX, Chen FB, et al. Diurnal neurobiological alterations after exposure to clozapine in first-episode schizophrenia patients. Psychoneuroendocrinology. 2016;64:108-116.

9. Chen PY, Chen $\mathrm{CH}$, Chang CK, et al. Orexin-a levels in relation to the risk of metabolic syndrome in patients with schizophrenia taking antipsychotics. Int $J$ Neuropsychopharmacol. 2019;22(1):28-36.

10. Liu Z, Zhang Y, Zhao T, et al. A higher body mass index in Chinese inpatients with chronic schizophrenia is associated with elevated plasma orexin-A levels and fewer negative symptoms. Nord J Psychiatry. 2020;74(7):525-532.

11. Misiak B, Bartoli F, Stramecki F, et al. Appetite regulating hormones in first-episode psychosis: a systematic review and meta-analysis. Neurosci Biobehav Rev. 2019;102:362-370.

12. Basoglu C, Oner O, Gunes C, et al. Plasma orexin A, ghrelin, cholecystokinin, visfatin, leptin and agouti-related protein levels during 6-week olanzapine treatment in first-episode male patients with psychosis. Int Clin Psychopharmacol. 2010;25(3):165-171.

13. Monda V, Salerno M, Sessa F, et al. Functional changes of orexinergic reaction to psychoactive substances. Mol Neurobiol. 2018;55(8):6362-6368.

14. Faesel N, Kolodziejczyk MH, Koch M, Fendt M. Orexin deficiency affects sociability and the acquisition, expression, and extinction of conditioned social fear. Brain Res. 2021;1751:147199.

15. Durairaja A, Fendt M. Orexin deficiency modulates cognitive flexibility in a sex-dependent manner. Genes Brain Behav. 2021;20(3):e12707.

16. Ochoa S, Usall J, Cobo J, Labad X, Kulkarni J. Gender differences in schizophrenia and first-episode psychosis: a comprehensive literature review. Schizophr Res Treatment. 2012;2012:916198.

17. Qin W, Liu C, Sodhi M, Lu H. Meta-analysis of sex differences in gene expression in schizophrenia. BMC Syst Biol. 2016;10(Suppl 1):9.

18. Markham JA. Sex steroids and schizophrenia. Rev Endocr Metab Disord. 2012;13(3):187-207.

19. Wang S, Zhao Y, Zhang L, et al. Stress and the brain: perceived stress mediates the impact of the superior frontal gyrus spontaneous activity on depressive symptoms in late adolescence. Hum Brain Mapp. 2019;40(17):4982-4993.

20. Tully LM, Lincoln SH, Liyanage-Don N, Hooker CI. Impaired cognitive control mediates the relationship between cortical thickness of the superior frontal gyrus and role functioning in schizophrenia. Schizophr Res. 2014;152(2-3):358-364.

21. Hof PR, Haroutunian V, Friedrich VL Jr, et al. Loss and altered spatial distribution of oligodendrocytes in the superior frontal gyrus in schizophrenia. Biol Psychiatry. 2003;53(12):1075-1085.

22. Gao B, Wang Y, Liu W, et al. Spontaneous activity associated with delusions of schizophrenia in the left medial superior frontal gyrus: a resting-state fMRI study. PLoS One. 2015;10(7):e0133766.

23. Braak H, Braak E. Neuropathological stageing of Alzheimerrelated changes. Acta Neuropathol. 1991;82(4):239-259.
24. Fronczek R, Overeem S, Lee SY, et al. Hypocretin (orexin) loss in Parkinson's disease. Brain. 2007;130(Pt 6):1577-1585.

25. Lu J, Zhao J, Balesar R, et al. Sexually dimorphic changes of hypocretin (Orexin) in depression. EBioMedicine. 2017; 18:311-319.

26. Zhao J, Bao AM, Qi XR, et al. Gene expression of GABA and glutamate pathway markers in the prefrontal cortex of non-suicidal elderly depressed patients. J Affect Disord. 2012;138(3):494-502.

27. Wang SS, Kamphuis W, Huitinga I, Zhou JN, Swaab DF. Gene expression analysis in the human hypothalamus in depression by laser microdissection and real-time PCR: the presence of multiple receptor imbalances. Mol Psychiatry. 2008;13(8):786-799, 741.

28. Kastin AJ, Akerstrom V. Orexin A but not orexin B rapidly enters brain from blood by simple diffusion. $J$ Pharmacol Exp Ther. 1999;289(1):219-223.

29. Strawn JR, Pyne-Geithman GJ, Ekhator NN, et al. Low cerebrospinal fluid and plasma orexin-A (hypocretin-1) concentrations in combat-related posttraumatic stress disorder. Psychoneuroendocrinology. 2010;35(7):1001-1007.

30. $\mathrm{Li} \mathrm{T}, \mathrm{Xu} \mathrm{W}$, Ouyang $\mathrm{J}$, et al. Orexin A alleviates neuroinflammation via OXR2/CaMKKbeta/AMPK signaling pathway after $\mathrm{ICH}$ in mice. $J$ Neuroinflammation. 2020;17(1):187.

31. Mediavilla C. [Orexin A as mediator in the gut-brain dialogue]. Rev Neurol. 2020;71(12):460-466.

32. Mediavilla C. Bidirectional gut-brain communication: A role for orexin-A. Neurochem Int. 2020;141:104882.

33. Nishino S, Ripley B, Mignot E, Benson KL, Zarcone VP. CSF hypocretin-1 levels in schizophrenics and controls: relationship to sleep architecture. Psychiatry Res. 2002;110(1):1-7.

34. Gerashchenko D, Murillo-Rodriguez E, Lin L, et al. Relationship between CSF hypocretin levels and hypocretin neuronal loss. Exp Neurol. 2003;184(2):1010-1016.

35. Karpiński P, Samochowiec J, Sąsiadek MM, Łaczmański Ł, Misiak B. Analysis of global gene expression at seven brain regions of patients with schizophrenia. Schizophr Res. 2020;223:119-127.

36. Li Q, Chen D, Liu T, et al. Sex differences in body mass index and obesity in chinese patients with chronic schizophrenia. $J$ Clin Psychopharmacol. 2016;36(6):643-648.

37. Abdellatif AA. The role of renin inhibition in treating the hypertensive patient with diabetes: a summary of preclinical and clinical evidence. Expert Rev Cardiovasc Ther. 2012;10(2):251-263.

38. Ray NJ, Brittain JS, Holland P, et al. The role of the subthalamic nucleus in response inhibition: evidence from local field potential recordings in the human subthalamic nucleus. Neuroimage. 2012;60(1):271-278.

39. Angermeyer MC, Kühn L, Goldstein JM. Gender and the course of schizophrenia: differences in treated outcomes. Schizophr Bull. 1990;16(2):293-307.

40. Xiang YT, Wang CY, Si TM, et al. Sex differences in use of psychotropic drugs and drug-induced side effects in schizophrenia patients: findings of the Research on Asia Psychotropic Prescription (REAP) studies. Aust $N Z J$ Psychiatry. 2011;45(3):193-198.

41. Lambe EK, Aghajanian GK. Hypocretin (orexin) induces calcium transients in single spines postsynaptic to identified thalamocortical boutons in prefrontal slice. Neuron. 2003;40(1):139-150. 
42. Lambe EK, Liu RJ, Aghajanian GK. Schizophrenia, hypocretin (orexin), and the thalamocortical activating system. Schizophr Bull. 2007;33(6):1284-1290.

43. Lis M, Stańczykiewicz B, Liśkiewicz P, Misiak B. Impaired hormonal regulation of appetite in schizophrenia: a narrative review dissecting intrinsic mechanisms and the effects of antipsychotics. Psychoneuroendocrinology. 2020;119:104744.

44. Burdakov D, Karnani MM, Gonzalez A. Lateral hypothalamus as a sensor-regulator in respiratory and metabolic control. Physiol Behav. 2013;121:117-124.

45. Grafe LA, Bhatnagar S. The contribution of orexins to sex differences in the stress response. Brain Res. 2020;1731:145893.

46. Panhelainen AE, Korpi ER. Evidence for a role of inhibition of orexinergic neurons in the anxiolytic and sedative effects of diazepam: a c-Fos study. Pharmacol Biochem Behav. 2012;101(1):115-124.

47. Dalal MA, Schuld A, Pollmächer T. Lower CSF orexin A (hypocretin-1) levels in patients with schizophrenia treated with haloperidol compared to unmedicated subjects. Mol Psychiatry. 2003;8(10):836-837.

48. Nollet M, Gaillard P, Minier F, Tanti A, Belzung C, Leman S. Activation of orexin neurons in dorsomedial/ perifornical hypothalamus and antidepressant reversal in a rodent model of depression. Neuropharmacology. 2011;61(1-2):336-346.

49. Chen Q, de Lecea L, Hu Z, Gao D. The hypocretin/orexin system: an increasingly important role in neuropsychiatry. Med Res Rev. 2015;35(1):152-197. 